\title{
Exponential and Laguerre Squeezed States for su(1,1) Algebra and Calogero-Sutherland Model
}

\author{
Hong-Chen Fu*and Ryu Sasaki ${ }^{\dagger}$ \\ Yukawa Institute for Theoretical Physics, Kyoto University Kyoto 606-01, Japan \\ July 7,2021
}

\begin{abstract}
A class of squeezed states for the $\mathrm{su}(1,1)$ algebra is found and expressed by the exponential and Laguerre-polynomial operators acting on the vacuum states. As a special case it is proved that the Perelomov's coherent state is a ladder-operator squeezed state and therefore a minimum uncertainty state. The theory is applied to the two-particle Calogero-Sutherland model. We find some new squeezed states and compared them with the classical trajectories. The connection with some su(1,1) quantum optical systems (amplitude-squared realization, Holstein-Primakoff realization, the two mode realization and a four mode realization) is also discussed.
\end{abstract}

PACS numbers: 03.65.-w, 02.20.-a, 42.50.-p

\section{Introduction}

Squeezed states become more and more interesting in the quantum optics [1] and gravitational wave detection[2]. It is well known that there are three definitions of the squeezed states and coherent states [3, 4, that is, (1) the displacement-operator acting on the vacuum states, (2) the eigenstates of the linear combination of creation and the annihilation operators and (3) the minimum uncertainty states. These three methods are equivalent only for the simplest harmonic oscillator system. The minimum uncertainty method works well for both the coherent and squeezed states for any symmetry systems [5, [6] and the ladder-operator squeezed states for general systems are described in [7]. Both methods are equivalent [7], namely, the eigenstates satisfying

$$
\left(\mu J^{-}+\nu J^{+}\right)|\beta\rangle=\beta|\beta\rangle,
$$

* JSPS Fellow. On leave of absence from Instituite of Theoretical Physics, Northeast Normal University, Changchun 130024, P.R.China. E-mail: hcfu@yukawa.kyoto-u.ac.jp

†Supported partially by the grant-in-aid for Scientific Research, Priority Area 231 "Infinite Analysis" and General Research (C) in Physics, Japan Ministry of Education. 
are the minimum uncertainty states [7]. Here $\mu$ and $\nu$ are complex constants satisfying $|\nu / \mu|<1, J^{-}$and its hermitian conjugate $J^{+}$are the lowering and raising operators, respectively. In a previous paper we have discussed the squeezed states of arbitrary densitydependent multiphoton systems and expressed the coherent states and squeezed vacua in the exponential displacement-operator form [8]

In this paper, we shall restrict ourselves to the $\mathrm{su}(1,1)$ system

$$
\left[J^{+}, J^{-}\right]=-2 J^{0}, \quad\left[J^{0}, J^{ \pm}\right]= \pm J^{ \pm},
$$

and its discrete representation

$$
\begin{aligned}
J^{+}|k, n\rangle & =\sqrt{(n+1)(2 k+n)}|k, n+1\rangle, \\
J^{-}|k, n\rangle & =\sqrt{n(2 k+n-1)}|k, n-1\rangle, \\
J^{0}|k, n\rangle & =(n+k)|k, n\rangle .
\end{aligned}
$$

Here $|k, n\rangle(n=0,1,2, \cdots)$ is the complete orthonormal basis and $k=\frac{1}{2}, 1, \frac{3}{2}, 2, \cdots$ is the Bargmann index labeling the irreducible representation $(k(k-1)$ is the value of Casimir operator). Motivated by Bergou et. al. [9], we first write $|\beta\rangle=D(\alpha) \| \beta\rangle(D(\alpha)$ is the Perelomov's displacement operator, see (2.2) ) and find that $\| \beta\rangle$ can be written as an exponential operator acting on the vacuum state. This exponential operator can be cut off in special cases and reduces to a Laguerre-polynomial form. In particular, as a special case, we prove that the Perelomov's coherent state is the ladder-operator squeezed state of $\mathrm{su}(1,1)$ and therefore a minimum uncertainty state. The connection of these squeezed states with the Perelomov's coherent states is also revealed.

Let us emphasize that the squeezed states obtained in this way appear in a number of physical systems [9, 10, 11, 12, 13] and in some cases, such as Raman processes, they are present while normal squeezing is not [14]. Squeezed states of this type are also useful in improving the accuracy of interferometric measurements.

Recently the Calogero-Sutherland (CS) model has attracted considerable interest [15]. The two-particle CS model enjoys the su(1,1) dynamic symmetry [16, 17] and its coherent states are investigated in [18]. So we can apply the theory developed in the Sec.2 to investigate the squeezed states. As a concrete example we consider the first-order Lagurre polynomial squeezed state and compare it with the classical trajectory and the Perelomov's coherent state. Some interesting features are found.

The theory is also applied to the $\mathrm{su}(1,1)$ optical systems, namely, the density-dependent Holstein-Primakoff (HP) system [19], amplitude-squared system, two-mode system and a 
four-mode system. The truncated states of these systems have already been discussed by several authors one by one [9, 10, 11, 12, 13]. Our approach presents a unified treatment. The truncated states are expressed by the Laguerre polynomials in a unified way. We believe that the results on the density-dependent HP system for arbitrary $k$ is new.

In appendix A we prove that the Perelomov's displacement operator $D(\alpha)$ is ill-defined for $|\alpha|>2$ for the discrete representation (1.3). This seems not noticed before. In this connection an additional remark on the exponentiation of the $W_{\infty}$ algebra is given. The $W_{\infty}$ algebra is an infinite dimensional Lie algebra which plays important roles in particle physics and solid state physics [20].

We use the notation

$$
\llbracket f(n) \rrbracket ! \equiv f(n) f(n-1) \cdots f(1), \quad \llbracket f(0) \rrbracket ! \equiv 1
$$

where $f(n)$ is a function defined for non-negative integers $n$. It is obvious that it is related to the gamma function when $f(n)$ is a linear function of $n, \llbracket A+n \rrbracket !=\Gamma(A+n+1) / \Gamma(A+1)$.

\section{General approach to su(1,1) algebra}

We start with the eigenvalue equation (1.1). The special cases $\nu=0$ and $\beta=0$ have already been investigated in a previous paper [8]. The eigenstates are the lowering-operator coherent states and squeezed vacua expressed in terms of an exponential operator acting on the vacuum state. It is not known, however, how to solve equation (1.1) in its full generality. We here follow the spirit of Bergou et. al. [9] and write $|\beta\rangle$ in the form

$$
|\beta\rangle \equiv D(\alpha) \| \beta\rangle
$$

where $D(\alpha)$ is the Perelomov's displacement operator

$$
D(\alpha)=\exp \left(\alpha J^{+}-\alpha^{*} J^{-}\right)
$$

and the parameter $\alpha$ will be specified later. Here we should note that the operator $D(\alpha)$ is ill-defined for $|\alpha|>2$ for the discrete representation (1.3) (a proof is given in Appendix A). By making use of the following relations $\left(\alpha=r e^{i \theta}\right)$

$$
D^{-1}(\alpha) J^{-} D(\alpha)=\cosh ^{2} r J^{-}+\sinh ^{2} r e^{2 i \theta} J^{+}+e^{i \theta} \sinh (2 r) J^{0}
$$

we obtain the equation for $\| \beta\rangle$

$$
\begin{gathered}
\left\{\sinh (2 r)\left[\nu e^{-i \theta}+\mu e^{i \theta}\right] J^{0}+\left[\nu \cosh ^{2} r+\mu e^{2 i \theta} \sinh ^{2} r\right] J^{+}\right. \\
\left.\left.\left.+\left[\mu \cosh ^{2} r+\nu e^{-2 i \theta} \sinh ^{2} r\right] J^{-}\right\} \| \beta\right\rangle=\beta \| \beta\right\rangle .
\end{gathered}
$$


To solve this equation we simplify it by canceling the term $J^{+}$. This is achieved by requiring

$$
e^{2 i \theta} \tanh ^{2} r=-\nu / \mu
$$

by which $r, \theta$ are determined for given values of $\mu$ and $\nu$. We note here that $|\nu / \mu|=$ $\tanh ^{2} r<1$, which is in accord with our previous assumption, and that if $\alpha$ satisfies (2.5), then so does $-\alpha$. Under the condition (2.5), Eq.(2.4) is simplified as

$$
\left.\left.\left[e^{i \theta} \sinh (2 r) J^{0}+\cosh (2 r) J^{-}\right] \| \beta^{\prime}\right\rangle=\beta^{\prime} \| \beta^{\prime}\right\rangle
$$

where $\beta^{\prime}=\cosh ^{2} r \beta / \mu$ and we denote $\left.\left.\| \beta\right\rangle \equiv \| \beta^{\prime}\right\rangle$, for simplicity.

To obtain the explicit form of $\left.\| \beta^{\prime}\right\rangle$, we expand it as

$$
\left.\| \beta^{\prime}\right\rangle=\sum_{n=0}^{\infty} C_{n}|k, n\rangle .
$$

Then, inserting (2.7) into (2.6) and using (1.3), we get the following recursion relation

$$
\cosh (2 r) \sqrt{(n+1)(2 k+n)} C_{n+1}=\left[\beta^{\prime}-e^{i \theta} \sinh (2 r)(k+n)\right] C_{n}
$$

which leads to

$$
C_{n}=\frac{\llbracket \beta^{\prime}-e^{i \theta} \sinh (2 r)(k+n-1) \rrbracket !}{(\cosh (2 r))^{n} \sqrt{n ! \llbracket n+2 k-1 \rrbracket !}} C_{0} .
$$

Therefore

$$
\begin{aligned}
\left.\| \beta^{\prime}\right\rangle & =C_{0} \sum_{n=0}^{\infty} \frac{\llbracket \beta^{\prime}-e^{i \theta} \sinh (2 r)(n+k-1) \rrbracket !}{(\cosh (2 r))^{n} \sqrt{n ! \llbracket n+2 k-1 \rrbracket !}}|k, n\rangle \\
& =C_{0} \sum_{n=0}^{\infty} \frac{\llbracket \beta^{\prime}-e^{i \theta} \sinh (2 r)(n+k-1) \rrbracket !}{(\cosh (2 r))^{n} n ! \llbracket n+2 k-1 \rrbracket !}\left(J^{+}\right)^{n}|k, 0\rangle .
\end{aligned}
$$

For convenience, we introduce the number operator $\mathcal{N}$ by

$$
\mathcal{N} \equiv J^{0}-k, \quad \mathcal{N}|k, n\rangle=n|k, n\rangle
$$

Then one can show that

$$
\begin{aligned}
& \mathcal{N} J^{+}=J^{+}(\mathcal{N}+1), \quad f(\mathcal{N}) J^{+}=J^{+} f(\mathcal{N}+1) \\
& \left(f(\mathcal{N}) J^{+}\right)^{n}=\left(J^{+}\right)^{n} f(\mathcal{N}+1) f(\mathcal{N}+2) \cdots f(\mathcal{N}+n)
\end{aligned}
$$

where $f$ is an arbitrary function of $\mathcal{N}$. Then as a key step, using Eq. (2.13) with

$$
f(\mathcal{N})=\frac{\beta^{\prime}-e^{i \theta} \sinh (2 r)(k+\mathcal{N}-1)}{\cosh (2 r)(2 k+\mathcal{N}-1)},
$$


the state $\left.\| \beta^{\prime}\right\rangle$ is finally written in the exponential form

$$
\begin{aligned}
\left.\| \beta^{\prime}\right\rangle & =C_{0} \sum_{n=0}^{\infty} \frac{1}{n !}\left(f(\mathcal{N}) J^{+}\right)^{n}|k, 0\rangle \\
& =C_{0} \exp \left(\frac{\beta^{\prime}-e^{i \theta} \sinh (2 r)(k+\mathcal{N}-1)}{\cosh (2 r)(2 k+\mathcal{N}-1)} J^{+}\right)|k, 0\rangle \\
& =C_{0} \exp \left(\frac{\beta^{\prime}-e^{i \theta} \sinh (2 r)\left(J^{0}-1\right)}{\cosh (2 r)\left(J^{0}+k-1\right)} J^{+}\right)|k, 0\rangle \equiv C_{0} E\left(\beta^{\prime}\right)|k, 0\rangle .
\end{aligned}
$$

So the squeezed state $|\beta\rangle$ is obtained as

$$
|\beta\rangle=C_{0} D(\alpha) E\left(\beta^{\prime}\right)|k, 0\rangle
$$

From (2.8) it is easy to derive that

$$
\lim _{n \rightarrow \infty}\left|\frac{C_{n+1}}{C_{n}}\right| \equiv|\xi|, \quad \xi \equiv-e^{i \theta} \tanh (2 r)
$$

For real $\theta$ and $r$, we always have $|\xi|<1$. Therefore the state $\left.\| \beta^{\prime}\right\rangle$ is normalizable.

Now we see some special cases.

Case 1. When $\left.\beta^{\prime}=-e^{i \theta} \sinh (2 r) k, \| \beta^{\prime}\right\rangle$ has a simple form

$$
\left.\| \beta^{\prime}\right\rangle=C_{0} e^{-e^{i \theta} \tanh (2 r) J^{+}}|k, 0\rangle \equiv C_{0} e^{\xi J^{+}}|k, 0\rangle,
$$

which, by making use of the formula (for $r<1$ )

$$
\exp \left(-2 \alpha J^{+}+2 \alpha^{*} J^{-}\right)|k, 0\rangle=\left(1-|\xi|^{2}\right)^{k} \exp \left(\xi J^{+}\right)|k, 0\rangle
$$

can be normalized as

$$
\left.\| \beta^{\prime}\right\rangle=\exp \left(-2 \alpha J^{+}+2 \alpha^{*} J^{-}\right)|k, 0\rangle \equiv D(-2 \alpha)|k, 0\rangle .
$$

So we finally obtain a surprising result

$$
|\beta\rangle=D(\alpha) D(-2 \alpha)|k, 0\rangle=D(-\alpha)|k, 0\rangle
$$

This is nothing but the displacement-operator coherent state of the su(1,1) algebra [16, 17], known as the Perelomov's coherent state. But in this paper we obtain it in a different and more natural way. From our formalism we conclude that

- the Perelomov's coherent state $D(-\alpha)|k, 0\rangle$ is a squeezed state in the sense of ladderoperator definition, namely, it is an eigenstate of equation (1.1) with eigenvalue $\beta=$ $2 e^{i \theta} \mu k \tanh (-r)$, and therefore 
- it is a minimum uncertainty state for $\mathrm{su}(1,1)$ algebra.

This observation seems not have appeared in the literature.

Case 2. The infinite series can be cut off for some special values of $\beta^{\prime}$. Suppose that $\beta^{\prime}=e^{i \theta} \sinh (2 r)(M+k)$, where $M$ is a non-negative integer. Then we have

$$
C_{n}= \begin{cases}0, & n>M, \\ (-\xi)^{n} \frac{M !}{\sqrt{n ! \llbracket 2 k+n-1 \rrbracket !}(M-n) !} C_{0}, & n \leq M .\end{cases}
$$

Therefore

$$
\left.\| \beta^{\prime}\right\rangle=C_{0} \sum_{n=0}^{M} \frac{1}{\llbracket 2 k+n-1 \rrbracket !}\left(\begin{array}{c}
M \\
M-n
\end{array}\right)(-1)^{n}\left(\xi J^{+}\right)^{n}|k, 0\rangle .
$$

From formula (2.13) it follows that

$$
\begin{aligned}
\left(\frac{\mathcal{N}}{\mathcal{N}+2 k-1} \xi J^{+}\right)^{n}|k, 0\rangle & =\left(\xi J^{+}\right)^{n} \frac{\mathcal{N}+1}{\mathcal{N}+2 k} \frac{\mathcal{N}+2}{\mathcal{N}+2 k+1} \cdots \frac{\mathcal{N}+n}{\mathcal{N}+2 k+n-1}|k, 0\rangle \\
& =\frac{n !}{\llbracket 2 k+n-1 \rrbracket !}\left(\xi J^{+}\right)^{n}|k, 0\rangle .
\end{aligned}
$$

So we can write (2.23) in the Laguerre polynomial form

$$
\left.\| \beta^{\prime}\right\rangle=C_{0} L_{M}\left(\xi \frac{\mathcal{N}}{\mathcal{N}+2 k-1} J^{+}\right)|k, 0\rangle \equiv C_{0} L_{M}\left(\xi \frac{J^{0}-k}{J^{0}+k-1} J^{+}\right)|k, 0\rangle,
$$

where

$$
L_{M}(x) \equiv \sum_{n=0}^{M} \frac{1}{n !}\left(\begin{array}{c}
M \\
M-n
\end{array}\right)(-1)^{n} x^{n} .
$$

Furthermore, if $k=1 / 2$, equation (2.25) reduces to

$$
\left.\| \beta^{\prime}\right\rangle=C_{0} L_{M}\left(\xi J^{+}\right)|k, 0\rangle .
$$

In the HP realization with $k=\frac{1}{2}$, this result was reported by Fan et. al. [12].

If $M=0$, then $\left.\| \beta^{\prime}\right\rangle \rightarrow|k, 0\rangle$ and therefore

$$
|\beta\rangle=D(\alpha)|k, 0\rangle
$$

which is also a Perelomov's coherent state. Here we in fact have proved that it is a minimum uncertainty state and a ladder-operator squeezed state with the eigenvalue $\beta=2 e^{i \theta} \mu k \tanh r$.

Here we would like to remark that all the Perelomov's coherent states $D(\alpha)|k, 0\rangle$ can be viewed as the ladder-operator squeezed states of $\mathrm{su}(1,1)$, namely, they are the eigenstates of the eigenvalue equation

$$
\left(J^{-}-e^{2 i \theta} \tanh ^{2}(r) J^{+}\right) D(\alpha)|k, 0\rangle=\left(2 e^{i \theta} k \tanh r\right) D(\alpha)|k, 0\rangle .
$$


This is achieved by interpreting Eq.(2.5) as a constraint equation for $\nu / \mu$, not for $\alpha$. This conclusion can also be directly proved by differentiating $D(\alpha)|k, 0\rangle$ with respect to $r$ (see Appendix B).

Recall that the squeezed states of the oscillator can be obtained by applying an operator (squeeze operator) on the coherent states. So we ask if the state $|\beta\rangle$ can be expressed in a form of an operator, say $\mathcal{E}(\beta)$, acting on the Perelomov's coherent state. The answer is affirmative. To see this, we start with Eqs.(2.10), namely,

$$
\begin{aligned}
\left.\| \beta^{\prime}\right\rangle & =\sum_{n=0}^{\infty} \widetilde{C}_{n}\left(J^{+}\right)^{n}|k, 0\rangle \\
\widetilde{C}_{n} & =\frac{\llbracket \beta^{\prime}-e^{i \theta} \sinh (2 r)(n+k-1) \rrbracket !}{(\cosh (2 r))^{n} n ! \llbracket n+2 k-1 \rrbracket !} C_{0} .
\end{aligned}
$$

Then we have

$$
|\beta\rangle=C_{0} D(\alpha) E\left(\beta^{\prime}\right)|k, 0\rangle=C_{0}\left[D(\alpha) E\left(\beta^{\prime}\right) D^{-1}(\alpha)\right] D(\alpha)|k, 0\rangle
$$

By making use of the hermitian conjugate of Eq.(2.3) and $\alpha \rightarrow-\alpha$ we obtain

$$
\begin{aligned}
|\beta\rangle & =\left[\sum_{n=0}^{\infty} \widetilde{C}_{n}\left(D(\alpha) J^{+} D^{-1}(\alpha)\right)^{n}\right] D(\alpha)|k, 0\rangle \\
& =\left[\sum_{n=0}^{\infty} \widetilde{C}_{n}\left(\cosh ^{2} r J^{+}+\sinh ^{2} r e^{-2 i \theta} J^{-}-e^{-i \theta} \sinh (2 r) J^{0}\right)^{n}\right] D(\alpha)|k, 0\rangle \\
& \equiv C_{0} \mathcal{E}\left(\beta^{\prime}\right) D(\alpha)|k, 0\rangle .
\end{aligned}
$$

However, unfortunately, the operator $\mathcal{E}\left(\beta^{\prime}\right)$ cannot be written in an exponential form. But it can be cut off in the case $\beta^{\prime}=e^{i \theta} \sinh (2 r)(M+k)$, where $M$ is a non-negative integer as before (write $\mathcal{E}(M, \alpha) \equiv \mathcal{E}\left(\beta^{\prime}\right)$ in this special case)

$$
\mathcal{E}(M, \alpha)=\sum_{n=0}^{M} \frac{(-\xi)^{n}}{\llbracket 2 k+n-1 \rrbracket !}\left(\begin{array}{c}
M \\
M-n
\end{array}\right)\left(\cosh ^{2} r J^{+}+\sinh ^{2} r e^{-2 i \theta} J^{-}-e^{-i \theta} \sinh (2 r) J^{0}\right)^{n} .
$$

When $M=0$, it reduces to the identity operator, and when $M=1$, it becomes

$$
\mathcal{E}(1, \alpha)=1-\frac{\xi}{2 k}\left(\cosh ^{2} r J^{+}+\sinh ^{2} r e^{-2 i \theta} J^{-}-e^{-i \theta} \sinh (2 r) J^{0}\right) .
$$

Eqs. (2.32),(2.33) establish the relationship between the squeezed states $|\beta\rangle$ and the Perelomov's coherent states. This is especially important in the case where the Perelomov's coherent states are already known. In the next section we shall consider such an example, the two-particle Calogero-Sutherland model. 


\section{Calogero-Sutherland Model}

\subsection{Summary: CS model and su(1,1) symmetry}

The CS model of two-particles reduces to the problem of a singular oscillator governed by the Hamiltonian

$$
H=-\frac{\hbar^{2}}{2 m} \frac{\mathrm{d}^{2}}{\mathrm{~d} X^{2}}+\frac{1}{2} m \omega^{2} X^{2}+\frac{g^{2}}{X^{2}}
$$

after removing the center-of-mass motion. In terms of dimensionless variables

$$
x=\left(\frac{m \omega}{\hbar}\right)^{1 / 2} X, \quad \mathcal{G}^{2}=\frac{m g^{2}}{\hbar^{2}}, \quad \mathcal{H}=\frac{1}{\hbar \omega} H
$$

the Hamiltonian can be rewritten as

$$
\mathcal{H}=-\frac{1}{2} \frac{\mathrm{d}^{2}}{\mathrm{~d} x^{2}}+\frac{1}{2} x^{2}+\frac{\mathcal{G}^{2}}{x^{2}}
$$

It is easy to verify that the operators 16

$$
J^{+}=\frac{1}{2}\left[\frac{1}{2}\left(x-\frac{\mathrm{d}}{\mathrm{d} x}\right)^{2}-\frac{\mathcal{G}^{2}}{x^{2}}\right], \quad J^{-}=\frac{1}{2}\left[\frac{1}{2}\left(x+\frac{\mathrm{d}}{\mathrm{d} x}\right)^{2}-\frac{\mathcal{G}^{2}}{x^{2}}\right], \quad J^{0}=\frac{\mathcal{H}}{2},
$$

satisfy the $\mathrm{su}(1,1)$ defining relations (1.2). Then one finds that $\mathcal{H}$ has discrete eigenvalues $E_{n}=2 n+E_{0}, n=0,1,2, \cdots$ and the corresponding eigenstates $\psi_{n}$ can be written

$$
\psi_{n} \propto\left(J^{+}\right)^{n} \psi_{0}
$$

where $\psi_{0}$ is defined by $J^{-} \psi_{0}=0$ and $\mathcal{H} \psi_{0}=E_{0} \psi_{0}=\left(\lambda+\frac{1}{2}\right) \psi_{0}$. The normalized $\psi_{n}$ 's are found to be

$$
\psi_{n}(x)=(-1)^{n}\left[\frac{2 \Gamma(n+1)}{\Gamma(n+\lambda+1 / 2)}\right]^{1 / 2} x^{\lambda} e^{-x^{2} / 2} L_{n}^{(\lambda-1 / 2)}\left(x^{2}\right),
$$

where $L_{n}^{(\alpha)}(x)$ is Laguerre polynomial and $\lambda \equiv \frac{1}{2}+\frac{1}{2} \sqrt{1+8 \mathcal{G}^{2}}$ satisfies $\lambda(\lambda-1)=2 \mathcal{G}^{2}$. These states form an orthonormal set in the interval $(0, \infty)$. The representation of the generators on these states is

$$
\begin{aligned}
J^{+} \psi_{n}(x) & =\sqrt{(n+1)(n+\lambda+1 / 2)} \psi_{n+1}(x), \\
J^{-} \psi_{n}(x) & =\sqrt{n(n+\lambda-1 / 2)} \psi_{n-1}(x), \\
J^{0} \psi_{n}(x) & =(n+\lambda / 2+1 / 4) \psi_{n}(x),
\end{aligned}
$$

which is nothing but the $k=\lambda / 2+1 / 4$ discrete representation of $\operatorname{su}(1,1)$ algebra. Therefore the theory developed in Sec.2 can be applied to study the squeezed states of the CS model. 


\subsection{Squeezed states}

The Perelomov's coherent state $D(\alpha)|k, 0\rangle \equiv \Psi_{2}(x)$ for CS model has already been explicitly given [18]

$$
\Psi_{2}(x)=\frac{\sqrt{2}}{\sqrt{\Gamma(\lambda+1 / 2)}}\left(\frac{1-|\zeta|^{2}}{(1+\zeta)^{2}}\right)^{k} x^{\lambda} \exp \left(y x^{2}\right)
$$

where

$$
\zeta=e^{i \theta} \tanh (r) \quad \text { or } \quad r=\frac{1}{2} \ln \frac{1+|\zeta|}{1-|\zeta|}, \quad y=-\frac{1}{2}\left(\frac{1-\zeta}{1+\zeta}\right)=-\frac{1}{2}\left(\frac{\cosh r-\sinh r e^{i \theta}}{\cosh r+\sinh r e^{i \theta}}\right) .
$$

So we can easily calculate the other squeezed states from Eqs.(2.32),(2.33),(2.34). Here we only consider the $M=1$ case. In this case, by making use of Eq.(2.34) and the realization of $\mathrm{su}(1,1)$ algebra in terms of differential operators (3.4), we can easily obtain

$$
\Psi_{2}^{(1)}(x) \equiv C_{0} \mathcal{E}(1, \alpha) \Psi_{2}(x)=C_{0}^{\prime}\left(A+\sinh (2 r) x^{2}\right) x^{\lambda} \exp \left(y x^{2}\right)
$$

where

$$
A=\left(\lambda+\frac{1}{2}\right)(\cos \theta-i \cosh (2 r) \sin \theta) .
$$

Then the distribution can be easily obtained as

$$
\begin{aligned}
& \left|\Psi_{2}^{(1)}(x)\right|^{2}=\left|C_{0}^{\prime}\right|^{2}\left(|A|^{2}+\left(A+A^{*}\right) \sinh (2 r) x^{2}+\sinh ^{2}(2 r) x^{4}\right) x^{2 \lambda} e^{-Y x^{2}} \\
& Y=-\left(y+y^{*}\right)=(\cosh (2 r)+\sinh (2 r) \cos \theta)^{-1} \\
& \left|C_{0}^{\prime}\right|^{2}=2\left(|A|^{2} \frac{\Gamma\left(\lambda+\frac{1}{2}\right)}{Y^{\lambda+\frac{1}{2}}}+\left(A+A^{*}\right) \sinh (2 r) \frac{\Gamma\left(\lambda+\frac{3}{2}\right)}{Y^{\lambda+\frac{3}{2}}}+\sinh ^{2}(2 r) \frac{\Gamma\left(\lambda+\frac{5}{2}\right)}{Y^{\lambda+\frac{5}{2}}}\right)^{-1}(3
\end{aligned}
$$

Now let us analyze this distribution and compare it with the classical trajectory [18 and $\left|\Psi_{2}(x)\right|^{2}$. Fig. $(1,2,3)$ and Fig. $(4,5,6)$ show $\left|\Psi_{2}^{(1)}(x)\right|^{2}$ (solid curve) and $\left|\Psi_{2}(x)\right|^{2}$ (broken curve) for various values of $|\zeta|$ (or $r$ ), $\theta$ and $\lambda$. In general, the $\left|\Psi_{2}^{(1)}(x)\right|^{2}$ has up to three peaks because the positions $x_{p}^{2}$ of the peak satisfy a cubic equation. In these figures, the abscissa is dimensionless distance $x$ of the two particles (see equation (3.2)) and the ordinate is the probabilities $\left|\Psi_{2}(x)\right|^{2}$ and $\left|\Psi_{2}^{(1)}(x)\right|^{2}$. The vertical line denotes the position of the classical trajectory. Some features of these graphics are as follows.

(1). The highest peak of $\left|\Psi_{2}^{(1)}(x)\right|^{2}$ becomes sharper and sharper as $\theta$ decreases from 0 to $-\pi$. The maximum width of the peak at $\theta=0$ is determined by $r$, as $\frac{1}{\sqrt{Y}} \sim e^{r}$. This property is shared by $\left|\Psi_{2}(x)\right|^{2}\left[18\right.$. Especially, near $\theta=-\pi,\left|\Psi_{2}^{(1)}(x)\right|^{2}$ changes rapidly.

(2). The highest peak of $\left|\Psi_{2}^{(1)}(x)\right|$ follows the classical trajectory better than the $\left|\Psi_{2}(x)\right|^{2}$ for $\theta$ close to $-\pi$ (see Fig.(3) (6)). This is especially pronounced for large $\mathcal{G}$. Fig.(3) shows that the peak position of $\left|\Psi_{2}^{(1)}(x)\right|^{2}$ is almost the same as the classical trajectory. 
(3). $\left|\Psi_{2}^{(1)}(x)\right|^{2}$ allows the multi-peak structure, while $\left|\Psi_{2}(x)\right|^{2}$ has only one peak. However, for large $\mathcal{G},\left|\Psi_{2}^{(1)}(x)\right|^{2}$ has also one peak (see Fig. $(1,2,3)$ ) only.

(4). Similarly with $\left|\Psi_{2}(x)\right|^{2},\left|\Psi_{2}^{(1)}(x)\right|^{2}$ follows the classical trajectory well for large $\mathcal{G}$.

Let us remark that the time evolution of the classical trajectory and the Perelomov's coherent states is relatively simple. It is described by the linear increase of the parameter $\theta$ : from $\theta=\theta_{0}$ at $t=0$ to $\theta=\theta_{0}+\omega t$ at time $t$. However, this is not the case for the squeezed states presented here.

\subsection{Discussion}

Before closing this section, let us mention that the states $\mathcal{E}(M, \alpha) \Psi_{2}(x)$ have the following form

$$
\mathcal{E}(M, \alpha) \Psi_{2}(x)=\left(A_{0}+A_{1} x^{2}+\cdots+A_{M} x^{2 M}\right) x^{\lambda} e^{y x^{2}}
$$

where $A_{j}$ are some complex numbers and $y$ is same as above. Then $\left|\mathcal{E}(M, \alpha) \Psi_{2}(x)\right|^{2}$ and the normalization constant can be obtained easily. It is easy to see that $\left|\mathcal{E}(M, \alpha) \Psi_{2}(x)\right|^{2}$ has, in general, up to $2 M+1$ peaks.

\section{Some su $(1,1)$ optical systems}

Many quantum optical systems enjoy the $\mathrm{su}(1,1)$ symmetry. For example, the densitydependent HP system, the amplitude-squared system, the two-mode systems and a fourmode system are proposed recently. Here we show that these systems can be treated by the formalism in Sec.2 in a unified way.

\subsection{Density-dependent HP realization}

The $\mathrm{su}(1,1)$ can be realized in terms of the single-mode electromagnetic field operators

$$
J^{+}=a^{\dagger} \sqrt{2 k+N}, \quad J^{-}=\sqrt{2 k+N} a, \quad J^{0}=k+N
$$

where $a^{\dagger}$, $a$, and $N=a^{\dagger} a$ are the creation, annihilation and number operators of a single mode electromagnetic field satisfying $\left[a, a^{\dagger}\right]=1$. This is the well-known HP realization of $\mathrm{su}(1,1)$ [19. On the Fock space $|n\rangle=\frac{\left(a^{\dagger}\right)^{n}}{\sqrt{n !}}|0\rangle$, we have

$$
\begin{aligned}
J^{+}|n\rangle & =\sqrt{(n+1)(2 k+n)}|n+1\rangle, \quad J^{-}|n\rangle=\sqrt{n(2 k+n-1)}|n-1\rangle, \\
J^{0}|n\rangle & =(n+k)|n\rangle .
\end{aligned}
$$


In comparison with Eqs.(1.3), we see that the HP realizations give rise to the discrete representation of $\mathrm{su}(1,1)$ on the usual Fock space. Therefore, by replacing the lowest-weight state $|k, 0\rangle$ by the vacuum state $|0\rangle$ of the Fock space, we recover all the results in the Sec.2. When $k=1 / 2$, the state (2.27) reduces to the one given by Fan et. al. [12].

\subsection{Amplitude squared realization}

The amplitude squared $\mathrm{su}(1,1)$ is realized by

$$
J^{+}=\frac{1}{2} a^{\dagger 2}, \quad J^{-}=\frac{1}{2} a^{2}, \quad J^{0}=\frac{1}{2}\left(N+\frac{1}{2}\right) .
$$

The representation on the usual Fock space is completely reducible and decomposes into a direct sum of two irreducible representations on the sectors $S_{0}$ and $S_{1}$

$$
\left.S_{j}=\operatorname{span}\{\| n\rangle_{j} \equiv|2 n+j\rangle \mid n=0,1,2, \cdots\right\}, \quad j=0,1 .
$$

Representations on $S_{j}$ can be written as

$$
\begin{aligned}
\left.J^{+} \| n\right\rangle_{j} & \left.=\sqrt{(n+1)\left(n+j+\frac{1}{2}\right)} \| n+1\right\rangle_{j}, \\
\left.J^{-} \| n\right\rangle_{j} & \left.=\sqrt{n\left(n+j-\frac{1}{2}\right)} \| n-1\right\rangle_{j}, \\
\left.J^{0} \| n\right\rangle_{j} & \left.=\left(n+\frac{j}{2}+\frac{1}{4}\right) \| n\right\rangle_{j},
\end{aligned}
$$

where we have used the relation

$$
\frac{1}{2} \sqrt{(2 n+j)(2 n+j-1)}=\sqrt{n\left(n+j-\frac{1}{2}\right)}, \quad \text { for } j=0,1 .
$$

We see that on the sector $S_{j}$ the representation (4.5) is just the $k=\frac{j}{2}+\frac{1}{4}$ discrete representation of $\mathrm{su}(1,1)$. Then, from section 2 , we immediately obtain

$$
\left.\left.\| \beta^{\prime}\right\rangle_{j}=C_{0} \exp \left\{\frac{\beta^{\prime}-e^{i \theta} \sinh (2 r)\left(J^{0}-1\right)}{\cosh (2 r)\left(J^{0}-\frac{3}{4}+\frac{j}{2}\right)} J^{+}\right\} \| 0\right\rangle_{j},
$$

which reduces to

$$
\left.|\beta\rangle_{j}=D(-\alpha) \| 0\right\rangle_{j}, \quad D(-\alpha)=\exp \left(-\frac{\alpha}{2} a^{\dagger 2}+\frac{\alpha^{*}}{2} a^{2}\right)
$$

in the case $\beta^{\prime}=-e^{i \theta} \sinh (2 r)\left(\frac{j}{2}+\frac{1}{4}\right)$, and to

$$
\left.\left.\| \beta^{\prime}\right\rangle_{j}=C_{0} L_{M}\left(\xi \frac{J^{0}-1}{J^{0}+\frac{j}{2}-\frac{3}{4}} J^{+}\right) \| 0\right\rangle_{j}
$$


in the case $\beta^{\prime}=e^{i \theta} \sinh (2 r)\left(M+\frac{j}{2}+\frac{1}{4}\right)$, and furthermore to

$$
\left.|\beta\rangle_{j}=D(\alpha) \| 0\right\rangle_{j}, \quad D(\alpha)=\exp \left(\frac{\alpha}{2} a^{\dagger 2}-\frac{\alpha^{*}}{2} a^{2}\right),
$$

when $M=0$.

From Eqs.4.8) and (4.10) we see $D( \pm \alpha)$ is just the squeeze operator of the single mode electromagnetic field, and therefore the states in the sector $S_{0}$

$$
D( \pm \alpha) \| 0\rangle_{0} \equiv D( \pm \alpha)|0\rangle
$$

give rise to the usual squeezed vacuum states. Therefore we see that the squeezed vacuum state of Weyl algebra can also be viewed as the ladder-operator squeezed state of the $\operatorname{su}(1,1)$ algebra.

In the paper [9], Bergou et. al. expressed the cut off states $\left.\| \beta^{\prime}\right\rangle$ in the Hermite polynomial form in the whole Fock space. This is because in this case the operator $\left(J^{ \pm}\right)^{\frac{1}{2}} \propto\left(a^{\dagger}, a\right)$ can be defined. Then using the connection between Laguerre and Hermite polynomials we can rewrite the Laguerre polynomial state in each sector as the Hermite polynomial state in the whole Fock space. Therefore we see that the Hermite state corresponds to the reducible representation and the Laguerre state to the irreducible representation.

\subsection{Two-mode realization}

Consider the two-mode photon operators

$$
J^{+}=a^{\dagger} b^{\dagger}, \quad J^{-}=a b, \quad J^{0}=\frac{1}{2}\left(N_{1}+N_{2}+1\right),
$$

where $N_{1}=a^{\dagger} a, N_{2}=b^{\dagger} b$. These three operators generate the su(1,1), too. The Fock space $\mathcal{F}$ of the two-mode states is decomposed into a direct sum of irreducible invariant subspaces $\mathcal{F}_{p}^{ \pm}$

$$
\begin{aligned}
& \mathcal{F}=\mathcal{F}_{0} \oplus \mathcal{F}_{1}^{ \pm} \oplus \cdots \oplus \mathcal{F}_{p}^{ \pm} \oplus \cdots, \\
& \left.\mathcal{F}_{p}^{+} \equiv \operatorname{span}\{\| n\rangle_{+p} \equiv|n, n+p\rangle \mid n=0,1,2, \cdots\right\}, \\
& \left.\mathcal{F}_{p}^{-} \equiv \operatorname{span}\{\| n\rangle_{-p} \equiv|n+p, n\rangle \mid n=0,1,2, \cdots\right\} .
\end{aligned}
$$

Representations $R_{p}^{ \pm}$on $\mathcal{F}_{p}^{+}$and $\mathcal{F}_{p}^{-}$are isomorphic and take the following form

$$
\begin{aligned}
\left.J^{+} \| n\right\rangle_{ \pm p} & =\sqrt{(n+1)(n+p+1)} \| n+1\rangle_{ \pm p} \\
\left.J^{-} \| n\right\rangle_{ \pm p} & =\sqrt{n(n+p)} \| n-1\rangle_{ \pm p} \\
\left.J^{0} \| n\right\rangle_{ \pm p} & \left.=\left(n+\frac{p+1}{2}\right) \| n\right\rangle_{ \pm p} .
\end{aligned}
$$


which are the representation (1.3) with $k=(p+1) / 2$. Then replacing $|k, 0\rangle$ by $\| 0\rangle_{ \pm p}$ and $k$ by $(p+1) / 2$, we obtain a class of squeezed states of two-mode systems. Among them we would like to mention the solution

$$
D( \pm \alpha) \| 0\rangle_{ \pm p}, \quad D( \pm \alpha) \equiv \exp \left( \pm \alpha a^{\dagger} b^{\dagger} \mp \alpha^{*} a b\right)
$$

which is nothing but the two-mode squeezed vacuum state proposed by Caves and Schumaker [21]. There they defined the two-mode squeezed states by applying the coherent displacement-operators $D_{1}(\delta)=\exp \left(\delta a^{\dagger}-\delta^{*} a\right)$ and $D_{2}(\delta)=\exp \left(\delta b^{\dagger}-\delta^{*} b\right)$ ( $\delta$ is a complex number) of each mode on the above squeezed vacuum. This squeezed vacuum is a minimum uncertainty and ladder-operator squeezed state of the $\mathrm{su}(1,1)$ algebra.

Let us remark that the cut off states of this system were also discussed in 10, 13. In particular, the form $J^{+}=a^{\dagger} b^{\dagger}$ enables us to express the cut off state as the two-variable Hermite polynomial form, as discussed in the paper [13]. This procedure is carried out in the whole Fock space, not in the irreducible invariant subspaces of the $\mathrm{su}(1,1)$.

\subsection{Four-mode system}

Consider the following generators obtained from the two two-mode $\mathrm{su}(1,1)$ algebras (we call them (a,b)-mode and (c,d)-mode for convenience) in the last subsection

$$
J^{+}=a^{\dagger} b^{\dagger}+c^{\dagger} d^{\dagger}, \quad J^{-}=a b+c d, \quad J^{0}=\frac{1}{2}\left(a^{\dagger} a+b^{\dagger} b+c^{\dagger} c+d^{\dagger} d+2\right) .
$$

They satisfy the su(1,1) algebra, too. To be more precise, Eqs.4.16) gives a Kronecker product of representations (4.14) of (a,b)- and (c,d)-mode $\mathrm{su}(1,1)$ algebras, denoted by $R_{p_{1}}^{ \pm}$ and $R_{p_{2}}^{ \pm}$, respectively, which could be decomposed into the direct sum of the irreducible representations. For example,

$$
R_{p_{1}}^{-} \otimes R_{p_{2}}^{-}=\sum_{P=p_{1}+p_{2}+1}^{\infty} R_{P}^{-} .
$$

The basis for the subspaces carrying the representation $R_{P}^{-}$can be obtained from those of $(\mathrm{a}, \mathrm{b})$ - and $(\mathrm{c}, \mathrm{d})$-modes in terms of the Clebsch-Gordan coefficients, which have been explicitly given in 22]. In particular, the vacuum state is given by

$$
\begin{aligned}
\left|0, n, p_{1}, p_{2}\right\rangle= & \left(\begin{array}{c}
2 n+p_{1}+p_{2} \\
n+p_{1}
\end{array}\right)^{1 / 2} \sum_{n_{1}}^{n}(-1)^{n_{1}}\left[\left(\begin{array}{c}
n \\
n_{1}
\end{array}\right)\left(\begin{array}{c}
n+p_{1}+p_{2} \\
n_{1}+p_{1}
\end{array}\right)\right]^{1 / 2} \\
& \times\left|n_{1}+p_{1}, n_{1}\right\rangle \otimes\left|n-n_{1}+p_{2}, n-n_{1}\right\rangle .
\end{aligned}
$$

Then the representation $R_{P}^{-}$is a standard discrete irreducible representation of $\operatorname{su}(1,1)$ in the form (4.14). So the exponential and cut off states can be discussed in the same way as in the last subsection. 


\section{Conclusion}

In this paper we have studied a class of the exponential and Laguerre polynomial squeezed states of the discrete representations of the $\mathrm{su}(1,1)$ Lie algebra. We have shown as an important result that the Perelomov's coherent states of the $\mathrm{su}(1,1)$ Lie algebra are at the same time the eigenstates of a proper linear combination of the raising and the lowering operators $J^{ \pm}$, namely, they are ladder-operator squeezed states and the minimum uncertainty states. The relationship between these states and the Perelomov's coherent states is also revealed.

Applying to the CS model, we find a class of new minimum uncertainty states starting from the Perelomov's coherent state. We present the cut off squeezed state with $M=1$, analyzed its properties and compare them with the classical trajectory and Perelomov's coherent states.

The approach in this paper presents a unified treatment of quantum optical $\mathrm{su}(1,1)$ systems. In particular, the cut off states are expressed unifiedly in terms of the Laguerre polynomial. The connection with Hermite polynomial representation [9, 12, 13] of these states is clarified.

It is a good challenge to investigate further the exponential and cut off states for $M>1$ for the CS model. The present approach is also expected to play important roles in studying the time-dependent singular oscillator systems [23].

\section{Appendix A. Convergence of the operator $D(\alpha)$}

The following theorem and corollaries [24] on the convergence of a power series is powerful and useful in this appendix.

Theorem 1 If a power series of $r, a(r)=\sum_{m=0}^{\infty} a_{m} r^{m}$ converges at $r=r_{0}$, then it converges for all values of $r$ in the circle $|r|<\left|r_{0}\right|$.

This can be rephrased as

Corollary 1 If a power series of $r, a(r)$ diverges at $r=r_{0}$, then it never converges outside of the circle $|r|>\left|r_{0}\right|$.

We mainly use the following

Corollary 2 If a subseries $b(r)$ of a power series $a(r)$ diverges at $r=r_{0}$, then the original power series $a(r)$ never converges outside of the circle $|r|>\left|r_{0}\right|$. 
This can be proved as follows: Supposing that $a(r)$ converges at a point $r=r_{1},\left|r_{1}\right|>\left|r_{0}\right|$, then $a(r)$ converges absolutely at $r=r_{0}$. Therefore its arbitrary subseries, including $b(r)$, converge at $r=r_{0}$, which is a contradiction.

Now we prove that the operator $D(\alpha)$ (see (2.2)) is ill-defined for $|\alpha|>2$ for the discrete representation (1.3). To do this we first consider the operator $e^{r\left(J^{+}+J^{-}\right)}$and take the expectation value

$$
\left\langle k, n\left|e^{r\left(J^{+}+J^{-}\right)}\right| k, n\right\rangle=\left\langle k, n\left|\left(\sum_{m=0}^{\infty} \frac{r^{m}}{m !}\left(J^{+}+J^{-}\right)^{m}\right)\right| k, n\right\rangle .
$$

Since only the even power terms are non-vanishing we get

$$
\left\langle k, n\left|e^{r\left(J^{+}+J^{-}\right)}\right| k, n\right\rangle=\sum_{m=0}^{\infty} \frac{r^{2 m}}{(2 m) !}\left\langle k, n\left|\left(J^{+}+J^{-}\right)^{2 m}\right| k, n\right\rangle .
$$

Among the $2^{2 m}$ terms in the expansion of $\left(J^{+}+J^{-}\right)^{2 m}$ we take the middle term $J^{-m} J^{+m}$ only and consider the following subseries of (A.1)

$$
b(r)=\sum_{m=0}^{\infty} \frac{r^{2 m}}{(2 m) !}\left\langle k, n\left|J^{-m} J^{+m}\right| k, n\right\rangle,
$$

which can be easily evaluated:

$$
b(r)=\sum_{m=0}^{\infty} r^{2 m} d_{m}, \quad d_{m}=\frac{(n+m) !(n+2 k+m-1) !}{(2 m) ! n !(n+2 k-1) !} .
$$

From this we can easily find that its radius of convergence $\rho$ is given by

$$
\rho^{2}=\lim _{m \rightarrow \infty}\left|\frac{d_{m}}{d_{m+1}}\right|=\lim _{m \rightarrow \infty}\left|\frac{(2 m+1)(2 m+2)}{(n+m+1)(n+2 k+m)}\right|=4 .
$$

Thus we find from Corollary 2 that the operator $e^{r\left(J^{+}+J^{-}\right)}$diverges for $|r|>2$.

Secondly, taking into account of the algebraic isomorphism of $\mathrm{su}(1,1)$

$$
J^{+} \longrightarrow e^{i\left(\theta+\frac{\pi}{2}\right)} J^{+}, \quad J^{-} \longrightarrow e^{-i\left(\theta+\frac{\pi}{2}\right)} J^{-}, \quad J^{0} \longrightarrow J^{0},
$$

and replacing $r \rightarrow-i r$, with real $r$, we find that the operator $D(\alpha)$ is ill-defined for $|\alpha|>2$, $\alpha=r e^{i \theta}$.

It is interesting that each exponential operator in the right side of the identity

$$
e^{\alpha J^{+}-\alpha^{*} J^{-}}=e^{\zeta J^{+}} e^{-\ln (\cosh r) 2 J^{0}} e^{-\zeta^{*} J^{-}}, \quad \zeta=e^{i \theta} \tanh (r),
$$

is well-defined for all real values of $r$ because $|\zeta|<1$ is always true. One might be tempted to use the above identity to define the left side $e^{\alpha J^{+}-\alpha^{*} J^{-}}$for all real value of $r$. However, in the proof of this identity, differentiation on the left-side is used [25]. That means the proof is 
valid only in the parameter range in which $D(\alpha)$ converges absolutely. Outside of this region the operator itself is ill-defined and the termwise differentiation is not allowed. A similar remark applies to the squeeze operator $e^{\frac{r}{2}\left(e^{i \theta} a^{\dagger 2}-e^{-i \theta} a^{2}\right)}$ of the simple-mode electromagnetic field 26

$$
e^{\frac{r}{2}\left(e^{i \theta} a^{\dagger 2}-e^{-i \theta} a^{2}\right)}=(\cosh r)^{-\frac{1}{2}} e^{\zeta \frac{1}{2} a^{\dagger 2}} e^{-\ln \cosh (r) N} e^{-\zeta^{*} \frac{1}{2} a^{2}}
$$

We believe the above formula is ill-defined for $|r|>2$.

By using the same argument we can show that the "unitary" operators $e^{i r\left(a^{\dagger n}+a^{n}\right)}, n \geq 3$, have zero radii of convergence. This fact has already been noted in paper [26]. The proof can be generalized further to any "unitary" operator $e^{i r h\left(a^{\dagger}, a\right)}$, in which $h\left(a^{\dagger}, a\right)$ is a hermitian operator consisting of a polynomial in $a^{\dagger}$ and $a$. If $h\left(a^{\dagger}, a\right)$ contains a term $f(N) a^{\dagger n}, n \geq 3$, where $f(N)$ is an arbitrary function of the number operator $N=a a^{\dagger}$, then the "unitary"

operator $e^{i r h\left(a^{\dagger}, a\right)}$ has a zero radius of convergence. These hermitian operators form an infinite dimensional Lie algebra, $W_{\infty}$ algebra, which is a symmetry algebra of the electron states of the lowest Landau level in a very strong magnetic field [20]. The above remark also implies that the $W_{\infty}$-group, to be obtained by the exponentiation of these $W_{\infty}$ generators, is simply ill-defined.

\section{Appendix B. Direct derivation of Eq.(22.29)}

Let us differentiate $D(\alpha)|k, 0\rangle\left(\alpha=r e^{i \theta}\right.$ as before) with respect to $r$

$$
\frac{\mathrm{d}}{\mathrm{d} r} D(\alpha)|k, 0\rangle=\left(e^{i \theta} J^{+}-e^{-i \theta} J^{-}\right) D(\alpha)|k, 0\rangle .
$$

On the other hand, $D(\alpha)|k, 0\rangle$ can be also expressed by using (A.6)

$$
D(\alpha)|k, 0\rangle=\frac{1}{(\cosh r)^{2 k}} \exp \left(e^{i \theta} \tanh r J^{+}\right)|k, 0\rangle .
$$

From this we get

$$
\frac{\mathrm{d}}{\mathrm{d} r} D(\alpha)|k, 0\rangle=\left(-2 k \tanh r+e^{i \theta} \operatorname{sech}^{2} r J^{+}\right) D(\alpha)|k, 0\rangle .
$$

By equating (B.1) and $(\underline{B .3})$ we get

$$
\left(e^{i \theta} J^{+}-e^{-i \theta} J^{-}\right) D(\alpha)|k, 0\rangle=\left(-2 k \tanh r+e^{i \theta} \operatorname{sech}^{2} r J^{+}\right) D(\alpha)|k, 0\rangle
$$

Multiplying (B.4) by $e^{i \theta}$ and moving the second term on the right side to the left, we immediately obtain the equation (2.29). 


\section{Acknowledgments}

H. C. Fu is grateful to Japan Society for Promotion of Science (JSPS) for the fellowship. He is also supported in part by the National Science Foundation of China.

\section{References}

[1] Workshop on squeezed states and Uncertainty Relations, edited by D. Han, Y.S. Kim and W.W.Zachary, NASA Conference Publication No.3135 (NASA, Washington DC, 1992).

[2] C. M. Caves, K.S. Thorne, R. W. P. Drever, V.D.Sandberg and M.Zimmerman, Rev. Mod. Phys. 52 (1980) 341.

[3] J. R. Klauder and B.S.Skagerstam, Coherent states-Applications in Physics and mathematical Physics (World Scientific, Singapore, 1985).

[4] W.-M. Zhang, D. H. Feng and R. Gilmore, Rev. Mod. Phys. 62 (1990) 867.

[5] M. M. Nieto and L. M.Simmons, Jr., Phys. Rev. Lett. 41 (1978) 207; Phys. Rev. D 20 (1979) 1321.

[6] M. M. Nieto and L. M. Simmons, Jr., Phys. Rev. D 20 (1979) 1332.

[7] M. M. Nieto and D. R. Truax, Phys. Rev. Lett. 71 (1993) 2843.

[8] H. C. Fu and R. Sasaki, Preprint YITP-95-2, quant-th/9510001.

[9] J. A. Bergou, M. Hillery and D. Yu, Phys. Rev. A 43 (1991) 515.

[10] C. C. Gerry and R. Grobe, Phys. Rev. A. 51 (1995) 4123.

[11] D. Yu and M. Hillery, Quantum Opt. 6 (1994) 37.

[12] H. Y. Fan, X. Ye and Z. H. Xu, Phys. Lett. A 199 (1995) 131.

[13] H. Y. Fan and X. Ye, Phys. Lett. A 175 (1993) 387.

[14] A. C. Chizhov, J. W. Haus and K. C. Yeong, Phys. Rev. A. 52 (1995) 1698.

[15] F. Calogero, J. Math. Phys. 10 (1969) 2191, 2197; 12 (1971) 419; B. Sutherland, J. Math. Phys. 12 (1971) 246, 251. 
[16] A. M. Perelemov, Generalized coherent states and their applications (Berlin: Springer, 1986).

[17] A. M. Perelemov, Sol. Phts. Usp. 20 (1977) 703; Commun. Math. Phys. 26 (1972) 222.

[18] G. S. Agarwal and S. Chaturvedi, J. Phys. A 28 (1995) 5747.

[19] T. Holstein and H. Primakoff, Phys. Rev. 58 (1940) 1098.

[20] B. Sakita, Phys. Lett. B315 (1993) 124, S. Iso, D. Karabali and B. Sakita, Phys. Lett. B296 (1992) 143.

[21] C. M. Caves and B. L. Schumaker, Phys. Rev. A 31 (1985) 3068.

[22] B. A. Bambah and G. S. Agarwal, Phys. Rev. A 51 (1995) 4918.

[23] J. K. Kim and S. P. Kim, preprint KNU-TH-16, 1994.

[24] E. T. Whittaker and G. N. Watson, A Course of Modern Analysis, (Cambridge Univ. Press, 1927), Page 30.

[25] D. R. Truax, Phys. Rev. D 31 (1985) 1989.

[26] R. A. Fisher, M. N. Nieto nd V.D. Sandberg, Phys. Rev. D 29 (1984) 1107. 


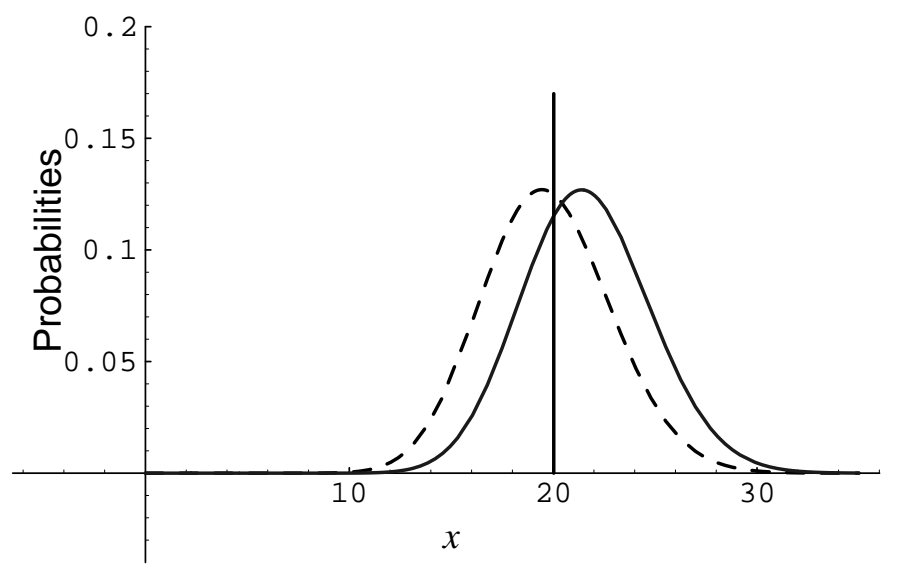

Figure 1: $\lambda=9.5, r=0.951, \theta=0$. Strong C-S coupling and broadly peaked.

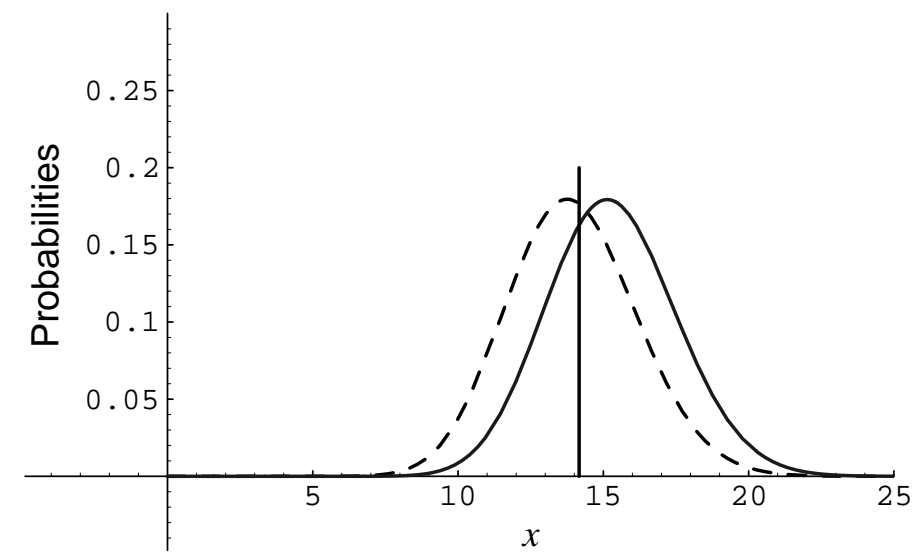

Figure 2: $\lambda=9.5, r=0.951, \theta=-\frac{\pi}{2}$. Strong coupling and mediumly peaked.

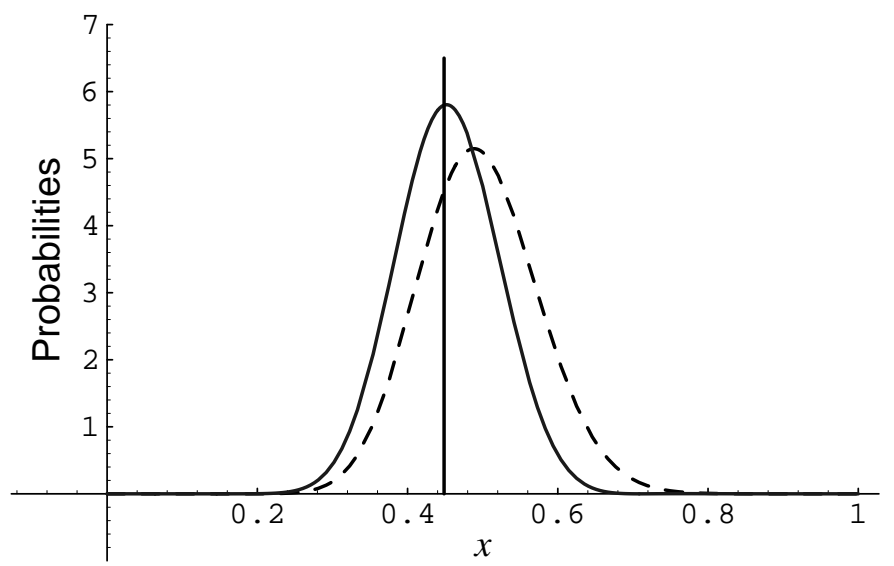

Figure 3: $\lambda=9.5, r=0.951, \theta=-\pi$. Strong coupling and narrowly peaked. 


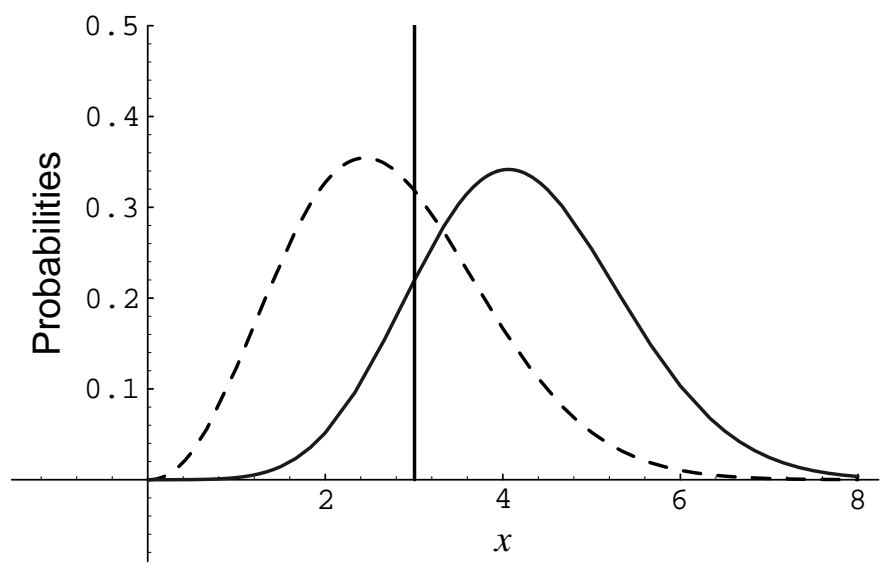

Figure 4: $\lambda=1.1, r=0.69, \theta=0$. Weak C-S coupling and broadly peaked.

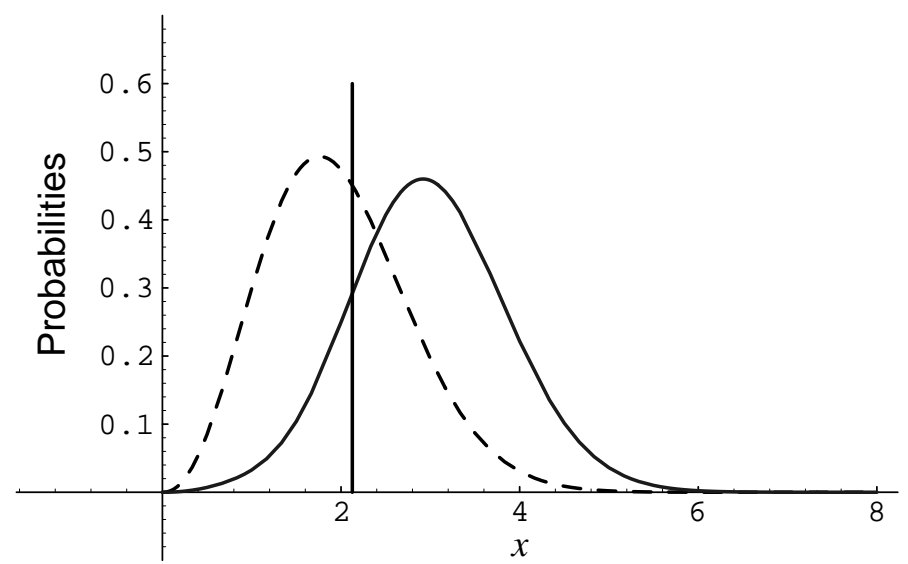

Figure 5: $\lambda=1.1, r=0.69, \theta=-\frac{\pi}{2}$. Weak coupling and mediumly peaked.

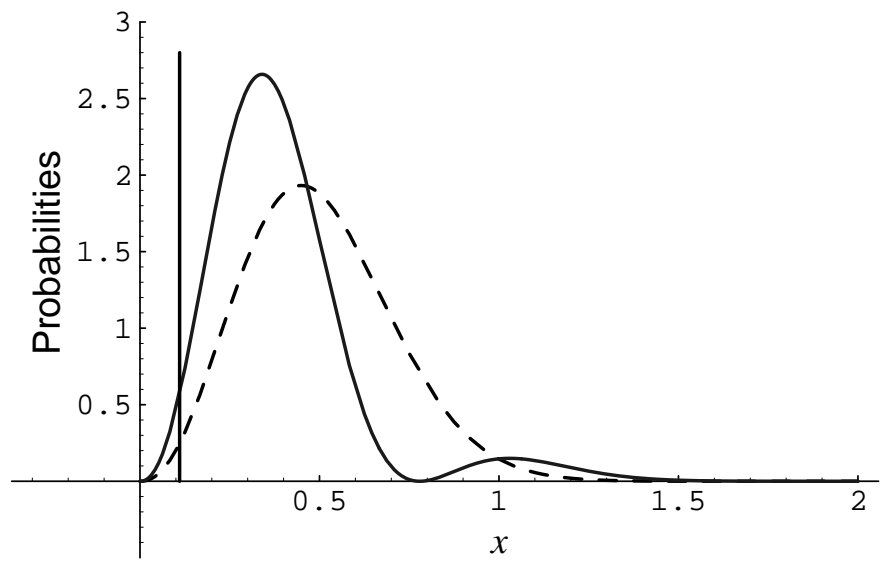

Figure 6: $\lambda=1.1, r=0.69, \theta=-\pi$. Weak coupling and narrowly peaked. 